\title{
Surveillance should be strengthened to improve epidemiological understandings of mosquito-borne Barmah Forest virus infection
}

\author{
Lutz Ehlkes, ${ }^{a}$ Keith Eastwood, ${ }^{a}$ Cameron Webbb and David Durrheim ${ }^{a}$ \\ Correspondence to Lutz Ehlkes (e-mail: ehlkes@bnitm.de).
}

Introduction: Barmah Forest virus (BFV) is a mosquito-borne virus causing epidemic polyarthritis in Australia. This study used case follow-up of cases from the surveillance system to demonstrate that routinely collected BFV notification data were an unreliable indicator of the true location of exposure.

Methods: BFV notifications from June 2001 to May 2011 were extracted from the New South Wales (NSW) Notifiable Conditions Information Management System to study case distribution. Disease cluster analysis was performed using spatial scan statistics. Exposure history data were collected from cases notified in 2010 and 2011 to accurately determine travel to high-risk areas.

Results: Cluster analysis using address data identified an area of increased BFV disease incidence in the mid-north coast of NSW contiguous with estuarine wetlands. When travel to this area was investigated, $96.7 \%(29 / 30)$ cases reported having visited coastal regions within four weeks of developing symptoms.

Discussion: Along the central NSW coastline, extensive wetlands occur in close proximity to populated areas. These wetlands provide ideal breeding habitats for a range of mosquito species implicated in the transmission of BFV. This is the first study to fully assess case exposure with findings suggesting that sporadic cases of BFV in people living further away from the coast do not reflect alternative exposure sites but are likely to result from travel to coastal regions. Spatial analysis by case address alone may lead to inaccurate understandings of the true distribution of arboviral diseases. Subsequently, this information has important implications for the collection of mosquito-borne disease surveillance information and public health response strategies.

$\mathrm{M}$ osquito-borne diseases are a growing concern in Australia, and an understanding of the spatial distribution of infection is required to refine surveillance strategies and public health interventions. Barmah Forest virus (BFV) disease is an arboviral disease endemic to Australia. ${ }^{1}$ The virus was isolated from mosquitoes in $1974^{2}$ and the first human cases were reported in $1986 .{ }^{3}$ The incubation period of BFV is probably seven to 10 days and symptoms of disease include rash, fever, arthralgia, myalgia and lethargy. ${ }^{4,5}$ $\mathrm{BFV}$ is a common cause of epidemic polyarthritis in Australia and carries important morbidity and economic impacts. It affects both genders and people of all ages. There is no specific treatment or vaccine available. ${ }^{6}$

New South Wales (NSW) uses a notifiable diseases register to record data on 57 communicable diseases and medical conditions. Case demographics are entered into an electronic database at sites across the state when standardized clinical and laboratory case definitions are met. For the majority of notifiable conditions, including BFV disease, ${ }^{7}$ the case definition is based on a suggestive clinical picture and confirmatory laboratory findings. It is mandatory for laboratories to report notifiable disease detections and convey available patient information to public health authorities. Routinely collected data include the patient's name, date of birth, sex, residential address and suspected date of disease onset. Owing to the large number of disease notifications and the nature of collection, data quality and completeness is adequate for monitoring disease trends but may be insufficient to allow detailed analysis of risk and exposure. To obtain this information for BFV disease it was necessary to conduct case follow-up interviews.

\footnotetext{
a Hunter New England Population Health, Newcastle, Australia.

Department of Medical Entomology, University of Sydney and Westmead Hospital, Westmead, Australia.

Submitted: 1 February 2012; Published: 2 August 2012

doi: 10.5365/wpsar.year.2012.3.1.004
} 
Seasonal BFV notifications in the Hunter New England Local Health District (HNELHD) of NSW (Figure 1) ranged from 60 to 173 between 2001 and 2011, with an average of 112 reports each season (incidence rate: 13.2 per 100 000).8,9

There is some uncertainty regarding the natural reservoirs of BFV in Australia. Although low levels of neutralizing antibodies have been detected in kangaroos, wallabies, possums, horses, cats and dogs after experimental infection, the detected viraemia is considered too low for an insect vector to acquire the virus. ${ }^{10,11}$ The genetic similarity of BFV strains across Australia, as well as the pace in which they spread, suggests an avian or bat host. ${ }^{12}$

There is a diverse range of mosquito species confirmed as vectors of BFV. The majority of important vector species, such as Aedes vigilax, Aedes procax, Aedes camptorhynchus and Verrallina funerea, are associated with either coastal estuarine wetlands (i.e. saltmarsh and mangrove habitats) or brackish water environments (i.e. tea-tree and paperbark swamps). ${ }^{13-15}$ However, some species associated with freshwater habitats and urban environments, such as Aedes notoscriptus, Culex annulirostris and Coquillettidia linealis, may also be involved. ${ }^{13,16,17}$ Given the diversity of potential vector species and differences in the environmental drivers of mosquito population abundance within the different habitats, it can be difficult to assess the regional health risks posed by BFV.

Although BFV disease has been documented in every state and territory of Australia, notifications occur predominantly on the Australian east coast, with sporadic inland cases. ${ }^{18,19}$ However, due to the limited knowledge of BFV's natural reservoir and a geographic distribution determined solely by the residential addresses of confirmed cases, the true distribution of the virus and thus the areas of risk are not definitively understood.

In this paper we aim to determine the likely risk exposure areas for BFV infection in the HNELHD of NSW, Australia by conducting structured interviews with all notified cases from June 2010 to May 2011 and to compare these identified risk exposure areas with those identified as having elevated standardized incidence rates when using routinely collected notification data from 2001 to 2010 .

\section{METHODS}

\section{Data acquisition}

Notification data consisting of primary demographic information were extracted from the NSW Notifiable Conditions Information Management System for the period June 2001 to May 2011 and analysed using

Figure 1. Location of the Hunter New England local health district within Australia

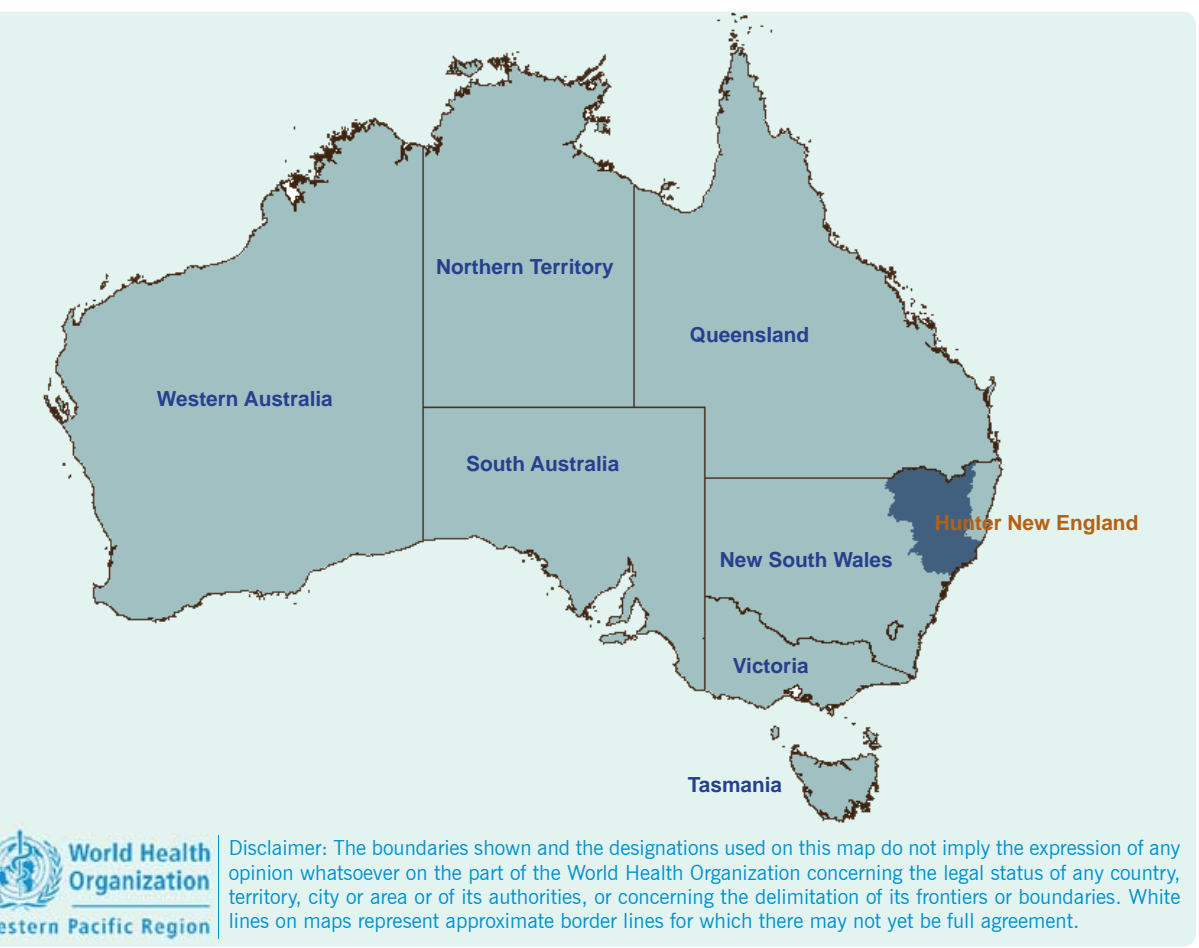


Maplnfo Professional version 10.0, a geographical information system.

Notification data were aggregated to local government area (LGA) level and the rates calculated, using population data obtained from the Australian Bureau of Statistics. Standardized incidence ratios (SIR) for each LGA were mapped to investigate the spatial distribution of BFV disease using the following formula:

SIR = observed notifications/expected
notifications
Observed notifications = notifications LGA/
population LGA
Expected notifications = notifications HNELHD/
population HNELHD
SIR = (notifications LGA/population LGA)/
(notifications HNELHD/population HNELHD)

\section{Mapping and spatial analysis}

The 1181 notifications between June 2001 and May 2011 were mapped to their residential address. Confidence intervals of $95 \%$ were applied to each SIR as the population in HNELHD is unevenly distributed. To identify disease clusters, incidence rates were calculated for each LGA, based on notification data and the 10-year seasonal mean of the underlying population. The data were analysed using Kulldorff's Circular Spatial Scan Statistics in SaTScan, version 8.0, using a purely spatial analysis with a discrete Poisson model and scanning for high incidence rates with a maximum cluster size of $50 \%$ of the population.

\section{Case interviews}

Cases notified in HNELHD area of NSW from June 2010 to May 2011 were interviewed by telephone about their travel history and possible exposure to mosquitoes during the incubation period of their infection (defined as four weeks).

\section{Ethics approval}

The Hunter New England Human Research Ethics Committee indicated that ethics approval was not required as this was regarded as a routine public health surveillance activity for a notifiable disease.

\section{RESULTS}

Spatial analysis of notifications from 2001 to 2011

Using routine notification data for the 10-year period, and applying 95\% confidence intervals, there were statistically increased SIRs in three LGAs: Port Stephens, Great Lakes and Greater Taree, all of which contain large coastal lakes (Figures $\mathbf{2}$ and $\mathbf{3}$ ).

The spatial analysis performed with SaTScan detected one significant cluster of notified BFV cases $(p$-value $<0.01)$. This cluster, with a radius of $109 \mathrm{~km}$, spanned an area of $37277 \mathrm{~km}^{2}$ and covered the LGAs of Greater Taree, Gloucester, Great Lakes, Walcha, Dungog and Port Stephens. SaTScan calculated the 10-year average as 61.0 annual notifications, compared to only 21.2 expected. This cluster showed a relative risk of 5.0, which means that people living in this area are five times as likely as the average population to contract BFV.

\section{Case follow-up}

Between June 2010 and May 2011, 62 BFV disease notifications were received for HNELHD. One case was accidentally reported twice. Contact details were available for 45 cases. The interviews revealed that two patients did not meet the case definition and were excluded. Ten cases could not be contacted and three refused to be interviewed. Thirty confirmed cases were interviewed, a 70\% response rate (30/43).

\section{Location of exposure}

Travel history obtained from respondents indicated 96.7\% (29/30) either lived in coastal LGAs $(n=22)$ or conducted outdoor activities in these areas during the four weeks before disease onset $(n=7)$. From those living in non-coastal areas, $87.5 \%(7 / 8)$ reported having been exposed to mosquitoes in coastal LGAs. The remaining case was unable to provide a connection with the coastal areas during the incubation period but admitted poor travel history recall.

\section{DISCUSSION}

In keeping with the indication from 10 years of routine notification data, the majority of BFV disease cases of 
Figure 2. Standardized incidence ratios of Barmah Forest virus per 100000 population by local governmental areas, with 95\% confidence interval ranges, Hunter New England local health district, 2001-2011

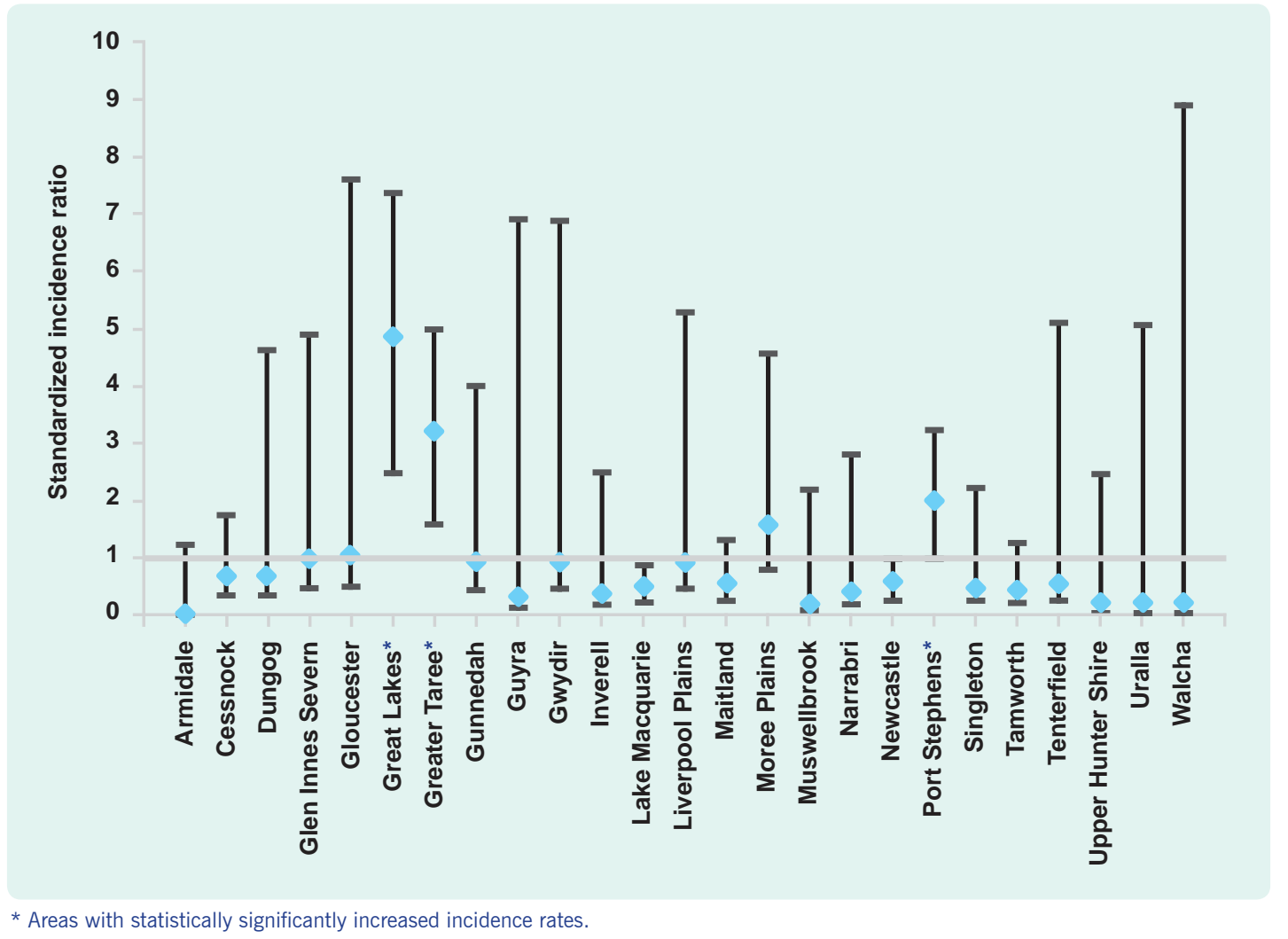

HNELHD in the 2010/2011 season were reported from the relatively densely populated central NSW coastal strip. While sporadic notifications were reported from inland areas, most of interviewed cases (96.7\%) either lived in coastal LGAs or reported spending time in these areas shortly before contracting BFV. This finding is reassuring, suggesting that there has not been an extension of the range of competent disease vectors inland. Some mosquito species found inland may transmit BFV, for example Culex annulirostris, which is associated with freshwater habitats. ${ }^{20}$ However, the abundance of this species is highly dependent on rainfall and, as a consequence, the local disease risk directly associated with this species may be variable. These results are in agreement with the findings of a recent Queensland study that used geostatistics to demonstrate higher incidence rates of BFV in coastal LGAs compared to inland areas. ${ }^{18}$

This is the first time a strengthened surveillance approach that investigated the true distribution of BFV has been reported. The current understanding of BFV distribution is based on the commonly observed coastal clustering of disease notifications, as well as the knowledge of distribution and competence of BFV vector species, but this has not explained inland cases. Studies that concentrate on the spatial allocation of notified cases using residential address data to plot incidence may misrepresent the true location of exposure and lead to an inaccurate understanding of the disease distribution and epidemiology. This may be particularly relevant to mosquito-borne diseases and other diseases that have vectors or intermediate hosts.

The results of this study have implications for future surveillance strategies and the communication of public health messages. The detection of abundant mosquito populations and/or the isolation of BFV (as well as other mosquito-borne pathogens) from collected specimens may trigger public health warnings promoting personal protection measures. This study suggests that these messages should be broadcast more widely than just to the coastal population, particularly during holiday periods when recreational travel to the coast may increase.

Serological diagnosis of BFV is subject to certain limitations including high false-positive rates and the need to confirm recent infection through collection of two serum specimens to demonstrate seroconversion. ${ }^{5}$ 
Figure 3. Standardized incidence ratios of Barmah Forest virus disease per 100000 population by local government area, Hunter New England local health district, 2001-2011

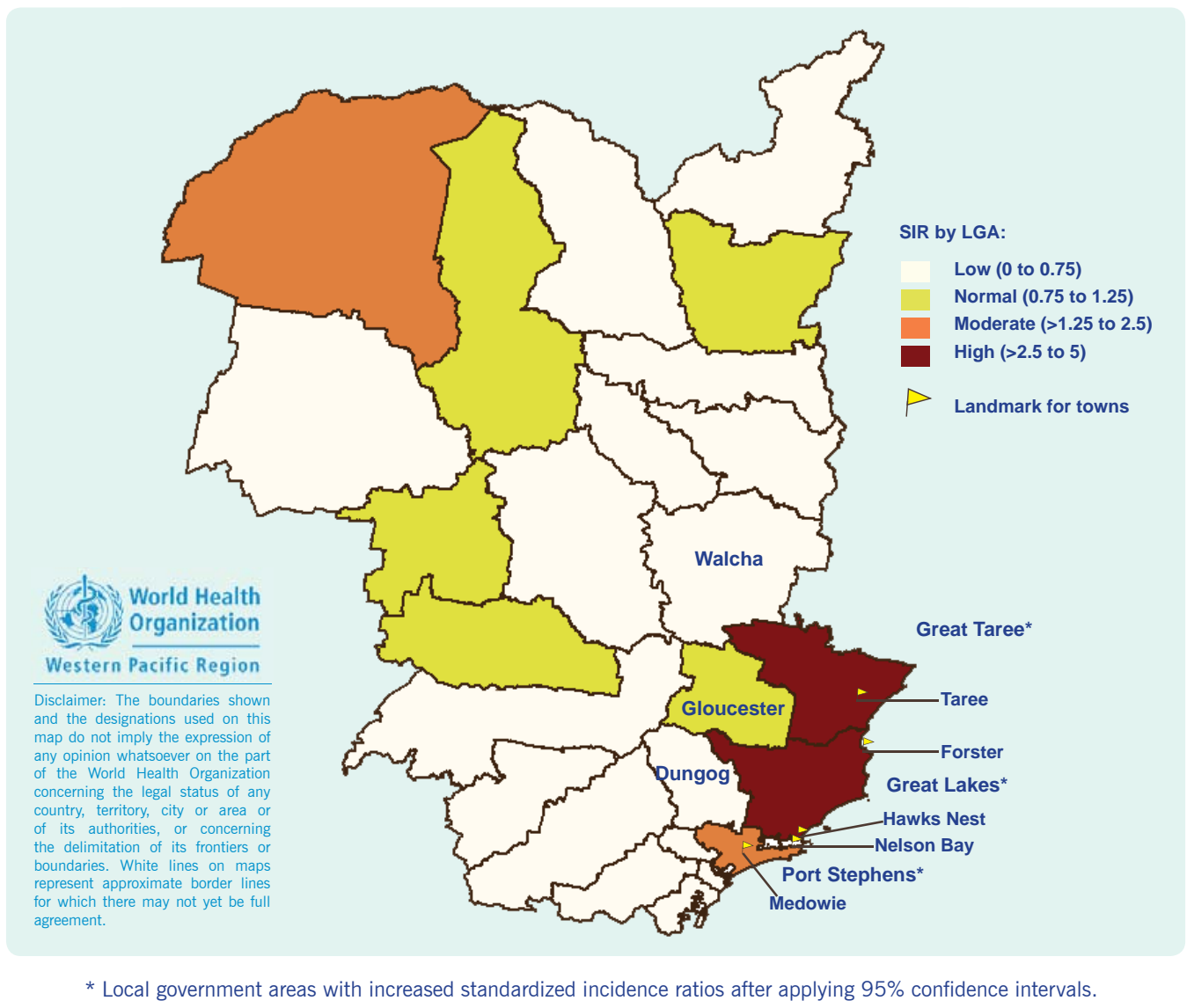

Confirmatory testing for IgM seroconversion is rarely conducted as only a single positive IgG test is necessary to be classified as a BFV case. This may compromise diagnostic certainty, and to limit this influence, the clinical features experienced by patients were carefully checked in addition to the laboratory diagnosis to ensure that their symptoms were compatible with a recent BFV infection.

The 2010-2011 season produced only 62 notifications, of which 30 patients were interviewed. The small sample size may impair general representativeness. We restricted our sample to those people who had been infected between June 2010 and May 2011 to reduce the possibility of recall bias. The majority of interviews were conducted within three months of the disease notification.

\section{CONCLUSION}

Geomapping in conjunction with spatial scan statistics using residential address data may be convenient for providing crude information on BFV disease clustering, but assuming that the home address approximates the site of exposure is fraught with problems. For diseases with an environmental association like mosquito-borne diseases, it may provide a false impression of the risk areas and epidemiology.

Telephone interviews confirmed that almost all recently notified BFV cases occurred in close proximity to estuarine wetlands and other coastal brackish water habitats, which are increasingly being favoured for housing development.

We suggest a revision of the arboviral surveillance system in Australia to include collection of information on travel history and risk exposure. Spatial surveillance that considers exposure location, as used in this study, may be of particular value for other vectorborne diseases where accurate information regarding viral transmission is lacking. Understanding the spatial patterns of infection rather than assuming coherence of case allocation and disease distribution may improve knowledge regarding the disease ecology and allow more targeted public health interventions. 


\section{Conflicts of interest}

None declared.

\section{Funding}

None.

\section{Acknowledgements}

The authors thank Dr Fakhrul Islam, Kerry Todd and Maggi Osbourn for the notification data extraction as well as Michelle Butler for statistical assistance. Lutz Ehlkes would like to thank Professor Thomas Kistemann for the supervision of his master's dissertation from which this manuscript is derived.

\section{References:}

1. Powers $\mathrm{AM}$ et al. Evolutionary relationships and systematics of the alphaviruses. Journal of Virology, 2001, 75: 10118-10131. doi:10.1128/JVI.75.21.10118-10131.2001 pmid: 11581380

2. Marshall ID, Woodroofe GM, Hirsch S. Viruses recovered from mosquitoes and wildlife serum collected in the Murray Valley of South-eastern Australia, February 1974, during an epidemic of encephalitis. The Australian Journal of Experimental Biology and Medical Science, 1982, 60:457-470. doi:10.1038/icb.1982.51 pmid:6299258

3. Vale TG et al. Human arbovirus infections along the south coast of New South Wales. The Australian Journal of Experimental Biology and Medical Science, 1986, 64:307-309. doi:10.1038/ icb.1986.32 pmid:3767767

4. Flexman JP et al. A comparison of the diseases caused by Ross River virus and Barmah Forest virus. The Medical Journal of Australia, 1998, 169:159-163. pmid:9734514

5. Cashman P et al. Barmah Forest virus serology; implications for diagnosis and public health action. Communicable Diseases Intelligence, 2008, 32:263-266. pmid:18767428

6. Smith DW, Speers DJ, Mackenzie JS. The viruses of Australia and the risk to tourists. Travel Medicine and Infectious Disease, 2010, 9:1-12. pmid:21679887

7. Arboviral diseases control guidelines for public health units. New South Wales Ministry of Health, 2004 (http:// www.health.nsw.gov.au/factsheets/guideline/arbo.html, accessed 13 June 2012).
8. Notifiable Conditions Information Management System. New South Wales Ministry of Health, Communicable Diseases Branch and Centre for Epidemiology and Research, 2012.

9. Health in Hunter New England Health eResource. New South Wales, Hunter New England Area Health Service, 2010 (http://www2.hnehealth.nsw.gov.au/HNEPH/HHNE/dem demHNELGApops.htm, accessed on 13 June 2012).

10. Kay $\mathrm{BH}$ et al. Mosquito feeding patterns and natural infection of vertebrates with Ross River and Barmah Forest viruses in Brisbane, Australia. The American Journal of Tropical Medicine and Hygiene, 2007, 76:417-423. pmid:17360861

11. Boyd AM et al. Experimental infection of Australian brushtail possums, Trichosurus vulpecula (Phalangeridae: Marsupialia), with Ross River and Barmah Forest viruses by use of a natural mosquito vector system. The American Journal of Tropical Medicine and Hygiene, 2001, 65:777-782. pmid:11791974

12. Poidinger $M$ et al. Genetic stability among temporally and geographically diverse isolates of Barmah Forest virus. The American Journal of Tropical Medicine and Hygiene, 1997, 57:230-234. pmid:9288821

13. Doggett SL et al. Barmah Forest virus epidemic on the south coast of New South Wales, Australia, 1994-1995: viruses, vectors, human cases, and environmental factors. Journal of Medical Entomology, 1999, 36:861-868. pmid:10593092

14. Ryan PA, Kay BH. Vector competence of mosquitoes (Diptera: Culicidae) from Maroochy Shire, Australia, for Barmah Forest virus. Journal of Medical Entomology, 1999, 36:856-860. pmid:10593091

15. Russell RC. Mosquitoes and mosquito-borne disease in southeastern Australia: a guide to the biology, relation to disease, surveillance, control and the identification of mosquitoes in south eastern Australia. Sydney, Westmead Hospital, 1993.

16. Jeffery JA et al. Vector competence of Coquillettidia linealis (Skuse) (Diptera: Culicidae) for Ross River and Barmah Forest viruses. Australian Journal of Entomology, 2002, 41:339-344. doi:10.1046/j.1440-6055.2002.00316.x

17. Passmore $J$ et al. An outbreak of Barmah Forest virus disease in Victoria. Communicable Diseases Intelligence, 2002, 26:600604. pmid: 12549534

18. Naish $S$ et al. Spatio-temporal patterns of Barmah Forest virus disease in Queensland, Australia. PLoS ONE, 2011, 6:e25688. doi:10.1371/journal.pone.0025688 pmid:22022430

19. Harvey L, Dwyer D. Recent increases in the notification of Barmah Forest virus infections in New South Wales. New South Wales Public Health Bulletin, 2004, 15:199-204. doi:10.1071/ NB04043 pmid:15711614

20. Doggett $S L$ et al. Arbovirus and vector surveillance in New South Wales, 2004/05-2007/08. Arbovirus Research in Australia, 2009, 10:28-37. 